\title{
Theoretical analysis of dynamic property for piezoelectric cantilever triple-layer benders with large piezoelectric and electromechanical coupling coefficients
}

\author{
Li Jiao Gong*, ${ }^{*}$, Cheng Liang Pan*, Qiao Sheng Pan ${ }^{\star}$ and Zhi Hua Feng ${ }^{\S}$ \\ *College of Mechanical and Electrical Engineering, \\ Shihezi University, Shihezi, Xinjiang 832003, P. R. China \\ ${ }^{\dagger}$ State Key Laboratory of Power Transmission Equipment \& System \\ Security and New Technology, Chongqing University \\ Chongqing 40044, P. R. China \\ tSchool of Instrument Science and Opto-Electronics Engineering \\ Hefei University of Technology, Hefei, Anhui 230009, P. R. China \\ $\S_{\text {China Department of Precision Machinery and Precision Instrumentation }}$ \\ University of Science and Technology of China, Hefei, Anhui 230026, P. R. China \\ iglj_mac@shzu.edu.cn.
}

Received 7 February 2016; Revised 6 April 2016; Accepted 4 May 2016; Published 15 June 2016

\begin{abstract}
Ferroelectric single crystals, such as PZN-PT, provide novel prospects in piezoelectric bending devices such as actuators, sensors or energy harvesters because of their extraordinarily large piezoelectric coefficients. However, large errors may occur in some analyses on electromechanical behaviors using the conventional models. We find the bending rigidity of piezoelectric composited bender is affected not only by thickness, width and the modulus of elasticity of the different layers but also electromechanical coupling coefficients (EMCCs) of the piezoelectric material and the larger EMCCs mean more marked effect. This paper focuses on the derivation of the applied input excitation and output response characteristics in the circular frequency domain for piezoelectric cantilever triple-layer benders (PCTBs), taking into account the secondary piezoelectric effect. Analytic dynamic descriptions of such actuators and transducers are obtained. Based on the presented models dynamic features of PCTB composed of PZN-8\%PT are calculated, and numerical results coincide with simulations using the finite element method (FEM).
\end{abstract}

Keywords: Dynamic property; electromechanical coupling (EMC); piezoelectric cantilever.

\section{Introduction}

In recent years, continuous effort has been exerted to develop novel solid state devices based on piezoelectric materials for abundant electromechanical applications by utilizing the positive or converse piezoelectric effect that can realize energy converting between mechanical energy and electric field energy. In some applications piezoelectric cantilever triplelayer benders (PCTBs) with one elastic nonpiezoelectric layer sandwiched between two piezoelectric layers are desirable because, with two piezoelectric layers, large displacement and generative force (as actuators) or high sensitivity (as sensors) can be expected and mechanical reliability can be improved $^{1}$ by using a nonpiezoelectric elastic layer.

There have been some research reports discussing the features and applications of piezoelectric bending mode element. ${ }^{1-13} \mathrm{Par}^{2}$ discussed the constitutive relations of piezoelectric benders. The constituent equations for unimorphs (i.e., heterogeneous bimorphs), which are made up of two different materials, for quasi-static cases have been determined ${ }^{3}$ by Smits et al. The dynamic admittance matrix of piezoelectric cantilever bimorphs was provided ${ }^{4}$ by Smits and Ballato in 1994. Afterwards, Lu and Lee also ${ }^{5}$ discussed and derived dynamic admittance matrix of piezoelectric cantilever bimorph. The constitutive relations of piezoelectric cantilever benders were discussed ${ }^{6,7}$ based on beam theory under the quasi-static equilibrium condition. However, the most detailed derivation of bimorph behavior as a function of frequency was presented ${ }^{4}$ by Smits et al. For symmetrical triple-layer piezoelectric bender static constitutive equations have been developed ${ }^{1}$ by Wang et al. in 1999; but the frequency response of PCTB is not described. Analytical distributed parameter solutions of cantilever piezoelectric triple-layer bender for energy harvesting were provided and discussed $^{8}$; however, frequency response described of PCTB was not sufficiently versatile. Tadmor ${ }^{12}$ pointed out electromechanical coupling between the electric field and the strain in the piezoelectric layer affected the effective moment of inertia of the piezoelectric layers, but no exact solution for PCTB was given. Ferroelectric single crystals, ${ }^{13-16}$ such as

This is an Open Access article published by World Scientific Publishing Company. It is distributed under the terms of the Creative Commons Attribution 4.0 (CC-BY) License. Further distribution of this work is permitted, provided the original work is properly cited. 
PZN-PT and PMN-PT, are constantly providing novel prospects in piezoelectric bending devices because of their extraordinarily large piezoelectric coefficients. To date, the dynamic admittance representation of symmetrical PCTB with large piezoelectric coefficient and electromechanical coupling coefficient (EMCC) has not been discussed in detail.

This paper is focused on the dynamic property determinations of symmetrical PCTB with arbitrary piezoelectric coefficients and EMCCs using procedures similar to those that Smits et al. used for the cantilever bimorph. These characteristics relate the harmonically varying excitation parameters, namely, an external tip force $F$, external moment $M$, and applied harmonic voltage $V$, to their response parameters, specifically the generated tip deflection $\delta$, tip angular slope $\theta$, and electric charge $Q$. The frequency response functions drawn of PCTB can also be used for better analysis of the behavior of PCTB with large EMCC as a function of frequency.

\section{Piezo Effect: Basic Constitutive Equations}

We chose the length direction of the bender as the $x$-axis, width as the $y$-axis, and height as the $z$-axis, as shown in Fig. 1. To obtain a relationship, the adherence of the glue is assumed to be great enough to prevent the three layers from slipping over each other. The piezoelectric material is poled along the height direction. In Fig. 1(a), the piezoelectric materials are connected electrically in series. In Fig. 1(b), the piezoelectric materials are connected in parallel. $l$ is the length of the bender, $h_{p}$ is the thickness of each piezoelectric slice, and $h_{m}$ is the thickness of the middle layer (Fig. 1(a)). Besides, $w$ means the width of the bender; $H$ is the total thickness of the bender.

The principal mechanical and electrical state variables (i.e., the electric field $E$, dielectric displacement $D$, mechanical stress $T$, and strain $S$ ) require directionality indexing. The directional parameters are given the subscripts 1,2 , and 3 , which correspond to the directions of $x, y$, and $z$, respectively. Mechanical shear stresses (couples) of $x, y$, and $z$, and the corresponding shear strains, are designated with the subscripts 4, 5, and 6, respectively. A linear constitutive law effectively models ${ }^{17}$ the interrelation among these variables; it sufficiently characterizes static and essential dynamic system designs. For the piezoelectric bender two cases must be considered, namely, plane stress and plane strain from the general constitutive equation of the piezoelectric effect in the hexagonal system, such as the poled lead zirconate titanate (PZT), $\mathrm{ZnO}$, and barium titanate. For plane stress condition, no stresses, except for $\mathrm{T}_{1}$, are assumed to exist, that is, $T_{2}=T_{3}=T_{4}=T_{5}=T_{6}=0, E_{1}=E_{2}=0$. For plane strain condition, we assume that no deformation is detected in the $y$-direction (i.e., $S_{2}=0$ ). In Fig. 1(a), the constitutive equation ${ }^{18}$ for the upper piezoelectric layer can be written in one-dimensional (1D) form as follows:

$$
\begin{aligned}
& S_{1}=s^{E} T_{1}+d E_{3}, \\
& D_{3}=d T_{1}+\varepsilon^{T} E_{3},
\end{aligned}
$$

where $\varepsilon^{T}$ is the free dielectric constant, $s^{E}$ is the elastic compliance at short circuit condition, and $d$ is the piezoelectric constant. Constants $s^{E}, d$, and $\varepsilon^{T}$ are respectively

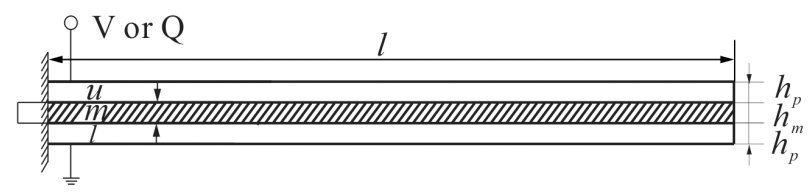

(a)

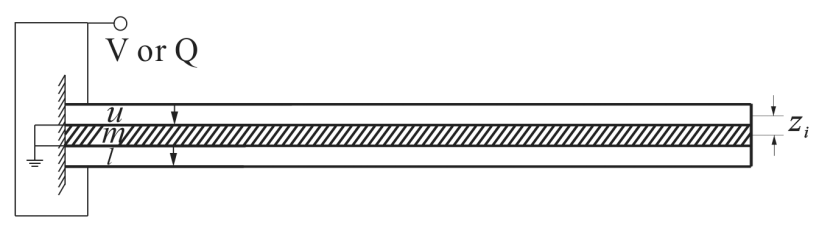

WIII

elastic non-piezoelectric layer

piezoelectric layer direction of polarization

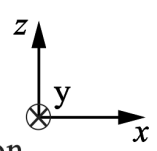

(b)

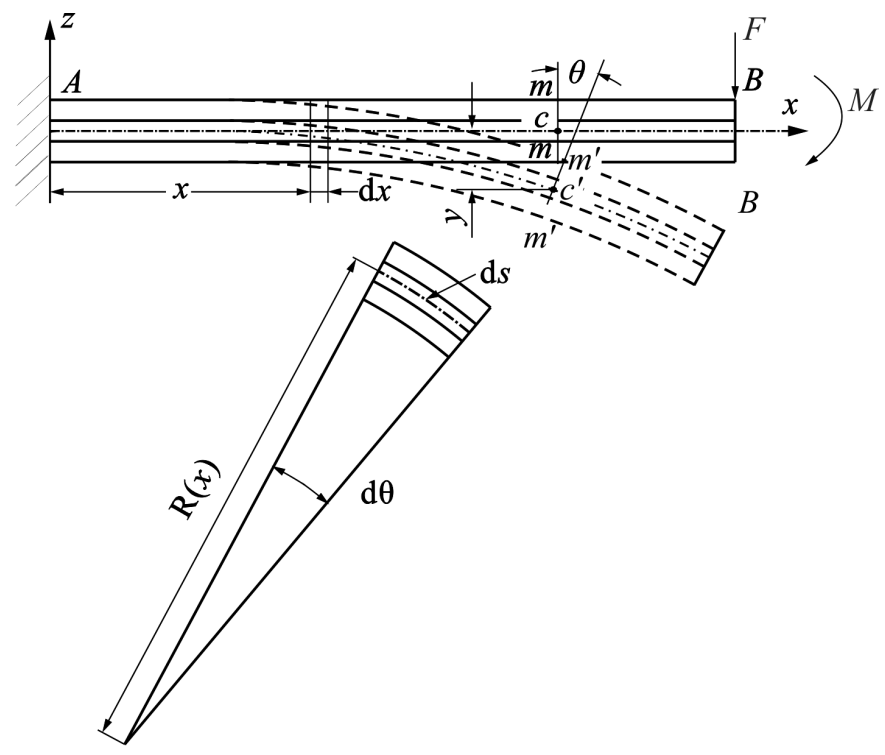

(c)

Fig. 1. (a) The piezoelectric materials are electrically in series connection; (b) the piezoelectric materials are electrically in parallel connection; (c) deformation of the cantilever-based bender. 
given $^{17}$ by

$$
\begin{gathered}
s^{E}= \begin{cases}s_{11}^{E} & \text { for plane stress, } \\
s_{11}^{E}-\frac{s_{12}^{E} s_{21}^{E}}{s_{22}^{E}} & \text { for plane strain, }\end{cases} \\
d= \begin{cases}d_{31} & \text { for plane stress, } \\
d_{31}-\frac{d_{32} s_{12}^{E}}{s_{22}^{E}} & \text { for plane strain, }\end{cases} \\
\varepsilon^{T}= \begin{cases}\varepsilon_{33}^{T} & \text { for plane stress } \\
\varepsilon_{33}^{T}-\frac{d_{32}^{2}}{s_{22}^{E}} & \text { for plane strain. }\end{cases}
\end{gathered}
$$

Piezoelectric elements convert mechanical strain energy into the electric field energy because of the piezoelectric effect. The piezoelectric bender works in dynamic deformation. The strain variation in the piezoelectric layers affects the electric field within layers. The electric field in the piezoelectric layers is generally assumed to be constant; this approximation is valid for piezoelectric elements with small electromechanical situation. However, several piezoelectric materials, such as PZN-PT, ${ }^{14-16}$ have large piezoelectric coefficients and EMCCs beyond small electromechanical situation. Tadmor et al. ${ }^{12}$ derived the change in effective field, and presented the strain effect on the electric field in the piezoelectric layer. $z(x, t)$ denotes instantly vertical displacement of the mass point from the mid-plane (Fig. 1(c)). This PCTB is symmetrical along the cross-section. The neutral surface lies on the middle surface of the bender. The motion of each point on the bender can be expressed in lateral displacement of the bender axis. Note that the axial bending strain $S_{1}(x, z)$ at a point of interest is a function of the radius of curvature $R(x, t)$ of the neutral axis and distance from that point to the neutral axis (i.e., the mid-plane of the triple-layer bender). Considering an element of the cantilever shown in Fig. 1(c), an extensional strain can be rewritten as follows:

$$
S_{1}(x, z)=-\frac{z}{R(x, t)} .
$$

The average strain $\bar{S}$ in the layer caused by the structural geometry is defined as follows:

$$
\bar{S}_{i}(x)=\frac{1}{h_{\text {layer }}} \int_{\text {layer }} S_{i}(x, z) d z=-\frac{z_{i}}{R(x, t)},
$$

where $z_{i}$ is the location of the center of the layer (Fig. 1(b)). For an electrical series connected PCTB, the form of $E_{3}(x, z)$ in the piezoelectric layers can be obtained by considering the secondary piezoelectric effect on the electric field as follows:

$$
\begin{aligned}
E_{3}(x, z) & =\frac{V}{2 h_{p}}+\frac{d}{\varepsilon^{T} s^{E}-d^{2}}\left[\bar{S}_{i}(x)-S_{1}(x, z)\right] \\
& =\frac{V}{2 h_{p}}+\frac{\xi}{d}\left[\bar{S}_{i}(x)-S_{1}(x, z)\right],
\end{aligned}
$$

where $\xi$ is given by $\xi=\frac{k_{31}^{2}}{1-k_{31}^{2}}$. The EMCC is $k_{31}^{2}=\frac{d^{2}}{s^{E} \varepsilon^{T}}$. The average strain is determined by Eq. (6).

The stress field within the piezoelectric material layer (Fig. 1(a)) after considering the secondary piezoelectric effect on the electric field may be determined as follows:

$$
T_{1}(x, z)=\frac{1}{s^{E}}\left[S_{1}(x, z)+\xi\left(S_{1}(x, z)-\bar{S}_{i}(x)\right)-\frac{d}{2 h_{p}} V\right] .
$$

If $\xi$ is much lower than 1 , the middle term is negligible relative to the extensional strain. The constitutive relation decreases to that obtained by Eq. (1a). Larger piezoelectric coefficients and EMCCs of piezoelectric materials have the greater impact than the secondary piezoelectric effect has on the electric field and the stress field within the piezoelectric material layer.

\section{Theoretical Analysis of the Dynamic Property}

In the following sections, discussions will proceed under the following external excitations: $F-$ an external tip force, $M$ - an external moment, and $V$ - a driving voltage. And the deflection, slope at the free end, and generated electric charge in the triple-layer bender are the vibration responses.

\subsection{Dynamic equations of the bending vibration}

For a bender's free vibration, the equation of motion ${ }^{19}$ can be written as

$$
\mathrm{EI} \frac{\partial^{4} z(x, t)}{\partial x^{4}}+\rho_{l}(x) \frac{\partial^{2} z(x, t)}{\partial t^{2}}=0,
$$

where EI is the flexural rigidity of the bender, $\rho_{l}$, is the mass per unit length of the bender. One method of solving Eq. (9) is the separation of variables. The solution can be represented by an absolutely and uniformly convergent series of the eigenfunctions, and it is assumed in the following form:

$$
z(x, t)=\sum_{i=1}^{N} Z_{i}(x) T_{i}(t)
$$

where $Z_{i}(x)$ and $T_{i}(t)$ are the mass normalized eigenfunction and the modal coordinate of the clamped-free beam for the $i$ th mode, respectively. For a simple harmonic motion, the symbolic time variation can be given by $T(t)=e^{j \omega t}(j=$ $\sqrt{-1}$ ). Therefore, the solution for Eq. (9) is

$$
\begin{aligned}
z(x, t)= & e^{j \omega t}(A \cosh \beta x+B \sinh \beta x \\
& +C \cos \beta x+D \sin \beta x),
\end{aligned}
$$

where $\beta^{4}=\rho_{l} \frac{\omega^{2}}{\text { EI }}$. Although this solution does not consider the external exciting force, the beam boundary conditions should be involved in solving (9). An external tip force $F$ and a bender subjected to an external moment $M$ (Fig. 1(c)) can both be used as the boundary conditions. Quantities $A, B, C$, 
and $D$ in (11) can be established from its boundary conditions for each problem.

Now the flexural rigidity of the PCTB needs to be analyzed. For a piezoelectric layer, the stress in length direction of a piezoelectric element is related to the strain and electric field by Eq. (8). For an elastic nonpiezoelectric layer, the stress is related to strain by Hooke's law and an extensional stress in the $x$-direction is given by

$$
T^{m}=\frac{1}{s^{m}} S(x, z)
$$

where $s^{m}$ is the compliance of the elastic middle layer.

For the elementary area is $w d z$, the elastic force $d F$ and the elementary moment $d M$ caused by the elastic force are $d M=z d F=z T_{1} w d z$. The integral resultant moment caused by stress can be calculated as follows:

$$
\begin{aligned}
M= & \int_{\frac{h_{m}}{2}}^{\frac{h_{m}}{2}+h_{p}} T^{u} w z d z+\int_{-\frac{h_{m}}{2}}^{\frac{h_{m}}{2}} T^{m} w z d z \\
& +\int_{-\left(\frac{h_{m}}{2}+h_{p}\right)}^{-\frac{h_{m}}{2}} T^{l} w z d z=\left[\frac{1}{s^{E}}\left(\frac{h_{m}^{2} h_{p}}{2}+h_{p}^{2} h_{m}+\frac{2 h_{p}^{3}}{3}\right)\right. \\
& \left.+\frac{h_{m}^{3}}{12} \frac{1}{s^{m}}+\frac{\xi h_{p}^{3}}{6 s^{E}}\right] \frac{w}{R(x, t)}-\frac{w\left(h_{p}+h_{m}\right) d}{2 s^{E}} V
\end{aligned}
$$

Assuming that the bending is small, the cantilever structure studied is vibrating in the $z$-direction. The inverse of the radius of curvature is the second derivative of the vertical displacement of the mid-plane $z(x, t)$ by the length $x$ or

$$
\frac{1}{R(x, t)}=\frac{d \theta}{d s} \approx \frac{d \theta}{d x}=\frac{\partial^{2} z(x, t)}{\partial x^{2}} .
$$

When no external electric field is applied on the piezoelectric triple-layer bender $(V=0)$, the resultant moment given by substituting Eq. (14) into (13) is reduced to

$$
\begin{aligned}
M= & {\left[\frac{w}{s^{E}}\left(\frac{h_{m}^{2} h_{p}}{2}+h_{p}^{2} h_{m}+\frac{2 h_{p}^{3}}{3}\right)+\frac{w h_{m}^{3}}{12 s^{m}}+\frac{\xi w h_{p}^{3}}{6 s^{E}}\right] } \\
& \cdot \frac{\partial^{2} z(x, t)}{\partial x^{2}} .
\end{aligned}
$$

Equation (15) states that the moment along the neutral axis of the bender is proportional to the dynamic radius of curvature of the bender. The scaling factor is the flexural rigidity of the PCTB. Thus, the flexural rigidity EI can be calculated as

$$
\mathrm{EI}=\frac{w}{s^{E}}\left(\frac{h_{m}^{2} h_{p}}{2}+h_{p}^{2} h_{m}+\frac{2 h_{p}^{3}}{3}\right)+\frac{w h_{m}^{3}}{12 s^{m}}+\frac{\xi w h_{p}^{3}}{6 s^{E}} .
$$

Equation (16) indicates that the larger EMCC of the piezoelectric material, the greater piezoelectric effect impact on the bending rigidity of a PCTB.

\subsection{The triple-layer bender subjected to an external tip force}

When a simple harmonic excitation lateral force $F=$ $F e^{j\left(\omega t+\varphi_{1}\right)}=F_{0} e^{j \omega t}$ is applied to the tip of the piezoelectric bender, the motion of the bender will be harmonic. In this case, the following boundary conditions are used to start the computation:

$$
\begin{gathered}
\left.z(x, t)\right|_{x=0}=0,\left.\quad \frac{\partial z(x, t)}{\partial x}\right|_{x=0}=0 \\
\left.\frac{\partial^{2} z(x, t)}{\partial x^{2}}\right|_{x=l}=0,\left.\quad \frac{\partial^{3} z(x, t)}{\partial x^{3}}\right|_{x=l}=\frac{F}{\mathrm{EI}} .
\end{gathered}
$$

By using these conditions to determine the constants $A, B, C$, and $D$ given in (11), we can obtain the following constants:

$$
\begin{aligned}
& -C=A=\frac{F(l)(\sinh \beta l+\sin \beta l)}{2 \beta^{3} \mathrm{EI}(1+\cosh \beta l \cos \beta l)}, \\
& -D=B=-\frac{F(l)(\cosh \beta l+\cos \beta l)}{2 \beta^{3} \mathrm{EI}(1+\cosh \beta l \cos \beta l)} .
\end{aligned}
$$

By inserting these values into (11), we can obtain the expression for the displacement of the beam in the transverse direction (z-direction) at each point along the $x$-axis as follows:

$$
\begin{aligned}
z(x, t)= & \frac{F(l)}{2 \beta^{3} \mathrm{EI}(1+\cosh \beta l \cos \beta l)} \\
& \times[(\sinh \beta l+\sin \beta l)(\cosh \beta x-\cos \beta x) \\
& +(\cosh \beta l+\cos \beta l)(\sin \beta x-\sinh \beta x)] .
\end{aligned}
$$

As shown in Fig. 1(c), the dynamic angle $\theta(x, t)$ subtended by the unit length is given by

$$
\theta(x, t) \approx \frac{\partial z(x, t)}{\partial x}
$$

Assumed that the deflections are small in these equations. This assumption is generally valid for piezoelectric benders, particularly those consisting of the piezoelectric single crystals, for example, PZN-PT, because the repeated large dynamic deflection can lead to piezoelectric layer breakage.

We obtain the deflection at the tip by substituting $x=l$ into (19) as follows:

$$
\begin{aligned}
\delta_{l l} & =\left.z(x, t)\right|_{x=l} \\
& =\frac{F(l)(\sin \beta l \cosh \beta l-\sinh \beta l \cos \beta l)}{\beta^{3} \mathrm{EI}(1+\cosh \beta l \cos \beta l)} .
\end{aligned}
$$

We get the slope $\theta$ at the tip as:

$$
\theta_{l l}=\left.\frac{\partial z(x, t)}{\partial x}\right|_{x=l}=\frac{F(l)}{\beta^{2} \mathrm{EI}}\left(\frac{\sin \beta l \sinh \beta l}{1+\cosh \beta l \cos \beta l}\right) .
$$

Then, we focus on the electric charge generated in the triple-layer bender. Given that no external electric field is applied on the piezoelectric bender $(V=0)$, the constitutive relations by introducing Eqs. (7) and (8) into Eq. (1b) are 
reduced to

$$
D_{3}=\frac{d}{s^{E}} \bar{S}_{1}(x),
$$

where directions 1 and 3 coincide with the $x$ - and $z$-axis, respectively.

The average strain $\bar{S}$ in the piezoelectric layer is expressed as Eq. (6). The electric charge $Q(t)$ collected by the electrodes can be obtained by integrating the electric displacement over the electrode area as

$$
Q=\int_{A} D_{3} d A=\int_{0}^{l} \frac{1}{2}\left(h_{p}+h_{m}\right) \frac{d}{s^{E}} \frac{\partial^{2} z(x, t)}{\partial x^{2}} w d x .
$$

By using the general form of the relative displacement given by Eq. (19) in Eq. (24) and by evaluating the spatial integral, we obtain

$$
Q=\frac{1}{2}\left(h_{p}+h_{m}\right) w \frac{d}{s^{E}} \frac{F(l)}{\beta^{2} \mathrm{EI}}\left(\frac{\sin \beta l \sinh \beta l}{1+\cosh \beta l \cos \beta l}\right) .
$$

\subsection{The triple-layer bender subjected to an external moment}

This section presents the derivation of the vibration responses resulting from the cantilevere-based piezoelectric bender subjected to an external harmonic moment excitation. In this case we get the boundary conditions as follows

$$
\begin{gathered}
\left.z(x, t)\right|_{x=0}=0,\left.\quad \frac{\partial z(x, t)}{\partial x}\right|_{x=0}=0 \\
\left.\frac{\partial^{2} z(x, t)}{\partial x^{2}}\right|_{x=l}=\frac{M(l)}{\mathrm{EI}},\left.\quad \frac{\partial^{3} z(x, t)}{\partial x^{3}}\right|_{x=l}=0 .
\end{gathered}
$$

By using these conditions as well as the constants $A, B, C$, and $D$ given in (11), we obtain

$$
\begin{aligned}
& -C=A=\frac{M(l)(\cosh \beta l+\cos \beta l)}{2 \beta^{2} \mathrm{EI}(1+\cosh \beta l \cos \beta l)}, \\
& -D=B=-\frac{M(l)(\sin \beta l-\sinh \beta l)}{2 \beta^{2} \mathrm{EI}(1+\cosh \beta l \cos \beta l)} .
\end{aligned}
$$

Substituting these values into (11), we obtain the solution for Eq. (9)

$$
\begin{aligned}
z(x, t)= & M(l) \times\left[\frac{(\cosh \beta l+\cos \beta l)(\cosh \beta x-\cos \beta x)}{2 \beta^{2} \mathrm{EI}(1+\cosh \beta l \cos \beta l)}\right. \\
& \left.+\frac{(\sin \beta l-\sinh \beta l)(\sinh \beta x-\sin \beta x)}{2 \beta^{2} \mathrm{EI}(1+\cosh \beta l \cos \beta l)}\right] .
\end{aligned}
$$

We derive the deflection at the tip by substituting $x=l$ into Eq. (28) as follows:

$$
\delta_{l l}=\left.z(x, t)\right|_{x=l}=\frac{M(l) \sin \beta l \sinh \beta l}{\beta^{2} \mathrm{EI}(1+\cosh \beta l \cos \beta l)} .
$$

We obtain the slope $\theta$ at the tip as follows:

$$
\theta_{l l}=M(l) \frac{(\sinh \beta l \cos \beta l+\sin \beta l \cosh \beta l)}{\beta \mathrm{EI}(1+\cosh \beta l \cos \beta l)} .
$$

By using the general form of the relative displacement given by (28) in (24) and by evaluating the spatial integral, the electric charge $Q(t)$ collected by the electrodes in this case can be represented by

$$
\begin{aligned}
Q= & \int_{A} D_{3} d A=\frac{1}{2}\left(h_{p}+h_{m}\right) w \frac{d}{s^{E}} \frac{M(l)}{\beta \mathrm{EI}} \\
& \times\left(\frac{\sinh \beta l \cos \beta l+\sin \beta l \cosh \beta l}{1+\cosh \beta l \cos \beta l}\right) .
\end{aligned}
$$

\subsection{The free triple-layer bender subjected to an electric voltage}

We now consider the case in which an AC voltage $V=V_{0} e^{j \omega t}$ is applied to the top and bottom electrodes of the triple-layer piezoelectric beam. We take two piezoelectric layers with a central elastic layer in a series connection, as shown in Fig. 1(a). One layer expands under the applied field, whereas the other contracts. We have obtained the moment of the PCTB, as shown in Eq. (13). The voltage $V$ does not vary with the length $x$ of the piezoelectric bender, so $\frac{\partial V}{\partial x}=0$. Differentiating Eq. (13) with respect to $x$, we have

$$
\frac{\partial M}{\partial x}=\mathrm{EI} \frac{\partial^{3} z(x, t)}{\partial x^{3}} .
$$

By combining a bender's static equilibrium position under its own weight and the torque balance equation at the centroid of the bender, the second-order small quantities can be ignored to obtain

$$
\mathrm{EI} \frac{\partial^{4} z(x, t)}{\partial x^{4}}=\rho_{l} \frac{\partial^{2} z(x, t)}{\partial t^{2}} .
$$

When a free PCTB is subjected to an electric voltage, the bending vibration equation is equivalent to the free vibration equation given earlier. The method of solving the equation is the same as that given in the former section, and the four boundary conditions for determining the constants are as follows:

$$
\begin{gathered}
\left.z(x, t)\right|_{x=0}=0,\left.\quad \frac{\partial z(x, t)}{\partial x}\right|_{x=0}=0 \\
\left.\frac{\partial^{2} z(x, t)}{\partial x^{2}}\right|_{x=l}=0,\left.\quad \frac{\partial^{3} z(x, t)}{\partial x^{3}}\right|_{x=l}=0 .
\end{gathered}
$$

Thus, the general solution for the relative transverse motion is given as

$$
\begin{aligned}
z(x, t)= & e^{j \omega t} A\left[(\cosh \beta x-\cos \beta x)-\frac{(\sinh \beta l-\sin \beta l)}{(\cosh \beta l+\cos \beta l)}\right. \\
& \times(\sinh \beta x-\sin \beta x)] .
\end{aligned}
$$


The initial condition is that the external moment is zero at the moving end. And after substituting Eq. (14) into Eq. (13) we obtain the second-order partial differential equation, as follows:

$$
\frac{\partial^{2} z(x, t)}{\partial x^{2}}=\frac{\left(h_{p}+h_{m}\right) d V}{2 s^{E} \mathrm{EI}}
$$

From (35) and (36), the number $A$ is expressed as

$$
A=\frac{\left(h_{p}+h_{m}\right) w d V}{4 \beta^{2} s^{E} \mathrm{EI}} \times \frac{(\cos \beta l+\cosh \beta l)}{(1+\cos \beta l \cosh \beta l)} .
$$

Thus, when the bender is driven with a dynamic voltage, the expression for instant vertical displacement of the mass point from the mid-plane in the triple-layer bender can be represented as

$$
\begin{aligned}
z(x, t)= & \frac{\left(h_{p}+h_{m}\right) w d V}{4 s^{E} \beta^{2} \mathrm{EI}(1+\cos \beta l \cosh \beta l)} \times\{(\cos \beta l+\cosh \beta l) \\
& \times(\cosh \beta x-\cos \beta x) \\
& -(\sinh \beta l-\sin \beta l)(\sinh \beta x-\sin \beta x)\} .
\end{aligned}
$$

We calculate the deflection at the tip under a harmonic voltage as follows:

$$
\delta_{l l}=\left.z(x, t)\right|_{x=l}=\frac{\left(h_{p}+h_{m}\right) w d V(\sin \beta l \sinh \beta l)}{4 s^{E} \beta^{2} \mathrm{EI}(1+\cos \beta l \cosh \beta l)} .
$$

By differentiating $z$ with respect to $x$ and by substituting $x=l$, we derive the slope $\theta$ under external excitation with an AC voltage as follows:

$$
\theta_{l l}=\frac{\left(h_{p}+h_{m}\right) w d V}{2 \beta E I s^{E}} \frac{(\cos \beta l \sinh \beta l+\cosh \beta l \sin \beta l)}{(1+\cos \beta l \cosh \beta l)} .
$$

Only external electric voltage is applied on the piezoelectric bender. By introducing Eq. (7), the constitutive relation leads to the following equation for the upper piezoelectric layer:

$$
D_{3}=\frac{d}{s^{E}} S_{1}(x, z)+\varepsilon^{T} \frac{V}{2 h_{p}}+\varepsilon^{T} \frac{\xi}{d}\left[\bar{S}_{i}(x)-S_{1}(x, z)\right] .
$$

Using the derivative of $z$ with respect to $x$, we obtain the second-order partial differential equation:

$$
\begin{aligned}
&\left(h_{p}+h_{m}\right) w d V\{(\cos \beta l+\cosh \beta l) \\
& \times(\cosh \beta x+\cos \beta x) \\
& \frac{\partial^{2} z(x, t)}{\partial x^{2}}=\frac{-(\sinh \beta l-\sin \beta l)(\sinh \beta x+\sin \beta x)\}}{4 \mathrm{EIs}^{E}(1+\cos \beta l \cosh \beta l)} .
\end{aligned}
$$

Considering that the charge in the electrodes is the integral over the surface of $D_{3}$. The charge $Q_{3}$ on the electrodes of the vibrator subjected to an electric voltage may be written as follows:

$$
Q_{3}=\frac{w l \varepsilon^{T} V}{2 h_{p}}\left(1-k_{31}^{2}+k_{31}^{2} \frac{w h_{p}\left(h_{p}+h_{m}\right)^{2} F(\beta l)}{4 s^{E} \mathrm{EI} \beta l}\right),
$$

where

$$
F(\beta l)=\frac{\cos \beta l \sinh \beta l+\cosh \beta l \sin \beta l}{1+\cos \beta l \cosh \beta l} .
$$

\subsection{The whole dynamic property and resonant frequency}

Now that we have these relations between the responses and excitations. The external excitations include an external tip force $F$, external moment $M$, and applied harmonic voltage $V$. The vibration response parameters of the piezoelectric bender consist of the tip deflection $\delta$, generated tip angular slope $\theta$, and electric charge $Q$. We should note that the PCTB considered in detail is connected in series, as shown in Fig. 1(a). In order to facilitate the further analysis, they can be collected in a $3 \times 3$ matrix form as follows:

$$
\left\{\begin{array}{l}
\delta \\
\theta \\
Q
\end{array}\right\}=\left[\begin{array}{ccc}
\frac{(\sin \beta l \cosh \beta l-\sinh \beta l \cos \beta l)}{\beta^{3} \mathrm{EI}(1+\cosh \beta l \cos \beta l)} & \frac{\sin \beta l \sinh \beta l}{\beta^{2} \mathrm{EI}(1+\cosh \beta l \cos \beta l)} & \frac{\left(h_{p}+h_{m}\right) w d(\sin \beta l \sinh \beta l)}{2 s^{E} \beta^{2} \mathrm{EI}(1+\cos \beta l \cosh \beta l)} \\
\frac{1}{\beta^{2} \mathrm{EI}}\left(\frac{\sin \beta l \sinh \beta l}{1+\cosh \beta l \cos \beta l}\right) & \frac{F(\beta l)}{\beta \mathrm{EI}} & \frac{\left(h_{p}+h_{m}\right) w}{2} \frac{d}{s^{E}} \frac{F(\beta l)}{\beta \mathrm{EI}} \\
\frac{\left(h_{p}+h_{m}\right) w d \sin \beta l \sinh \beta l}{2 s^{E} \beta^{2} \mathrm{EI}(1+\cosh \beta l \cos \beta l)} & \frac{\left(h_{p}+h_{m}\right) w}{2} \frac{d}{s^{E}} \frac{F(\beta l)}{\beta \mathrm{EI}} & \frac{w l \varepsilon^{T}}{2 h_{p}}\left(1-k_{31}^{2}+\frac{k_{31}^{2} w h_{p}\left(h_{p}+h_{m}\right)^{2} F(\beta l)}{4 s^{E} \mathrm{EI} \beta l}\right)
\end{array}\right]\left\{\begin{array}{l}
F \\
M \\
V
\end{array}\right\} .
$$

The first column of the matrix is $Y_{11}, Y_{21}$, and $Y_{31}$, and its entries can be derived from Eqs. (21), (22), and (25), respectively. The elements of the second column, namely, $Y_{12}, Y_{22}$, and $Y_{32}$, can be determined using Eqs. (29)-(31), respectively. For the third column, $Y_{13}, Y_{23}$, and $Y_{33}$ are calculated using Eqs. (39)-(41), respectively.
The piezoelectric materials are connected in parallel in Fig. 1(b). And the relations between the responses and excitations of the triple-layer bender in parallel connection were also derived. All the final relationships can be presented in the matrix (Eq. (46)). They are presented in the 
following $3 \times 3$ matrix:

$$
\left\{\begin{array}{l}
\delta \\
\theta \\
Q
\end{array}\right\}=\left[\begin{array}{ccc}
\frac{(\sin \beta l \cosh \beta l-\sinh \beta l \cos \beta l)}{\beta^{3} \mathrm{EI}(1+\cosh \beta l \cos \beta l)} & \frac{\sin \beta l \sinh \beta l}{\beta^{2} \mathrm{EI}(1+\cosh \beta l \cos \beta l)} & \frac{\left(h_{p}+h_{m}\right) w d(\sin \beta l \sinh \beta l)}{s^{E} \beta^{2} \mathrm{EI}(1+\cos \beta l \cosh \beta l)} \\
\frac{1}{\beta^{2} \mathrm{EI}}\left(\frac{\sin \beta l \sinh \beta l}{1+\cosh \beta l \cos \beta l}\right) & \frac{F(\beta l)}{\beta \mathrm{EI}} & \left(h_{p}+h_{m}\right) w \frac{d}{s^{E}} \frac{F(\beta l)}{\beta \mathrm{EI}} \\
\frac{\left(h_{p}+h_{m}\right) w d \sin \beta l \sinh \beta l}{s^{E} \beta^{2} \mathrm{EI}(1+\cosh \beta l \cos \beta l)} & \left(h_{p}+h_{m}\right) w \frac{d}{s^{E}} \frac{F(\beta l)}{\beta \mathrm{EI}} & \frac{w l \varepsilon^{T}}{2 h_{p}}\left(1-k_{31}^{2}+\frac{k_{31}^{2} w h_{p}\left(h_{p}+h_{m}\right)^{2} F(\beta l)}{4 s^{E} \mathrm{EI} \beta l}\right)
\end{array}\right]\left\{\begin{array}{l}
F \\
M \\
V
\end{array}\right\}
$$

Let the denominator of the elements in Eqs. (45) and (46) be equal to zero. Thus, the characteristic equation is derived

$$
1+\cos \beta l \cosh \beta l=0 .
$$

We then calculate the eigenvalues of the characteristic equation by a number of values of $\beta_{i} l$, which corresponds to each normal mode of the bending vibration. Values of $\beta_{i} l$ for the first four flexural vibrations are 1.8751, 4.6941, 7.8548, and 10.9955 , respectively. These values are then fed into the natural frequency calculation to obtain the natural frequencies. The resonant frequencies of the bending vibration for the PCTB are found to be

$$
\begin{gathered}
\omega_{n}=\frac{\left(\beta_{n} l\right)^{2}}{l^{2}} \sqrt{\frac{\left(6 h_{m}^{2} h_{p}+12 h_{p}^{2} h_{m}+8 h_{p}^{3}+2 \xi h_{p}^{3}\right)+\eta h_{m}^{3}}{12 s^{E}\left(2 \rho_{p} h_{p}+\rho_{m} h_{m}\right)}}, \\
n=1,2,3, \ldots,
\end{gathered}
$$

where we define $\eta=E_{m} / E_{p}, E_{m}$ is the Young's modulus of the middle layer, $E_{p}$ is the Young's modulus of the piezoelectric layer.

\section{Applications and Discussion}

With the help of MATLAB, the results of the established analytic model of the PCTB in series connection are investigated and predicted. The commercial finite element program ANSYS is also employed to perform the computation. Figure 2 shows the first mode shape of the PCTB. Twentynode element solid 226 is used for the model of PZN-8\%PT, and solid 95 is used for the copper model. The results indicate that the findings of the analytic model are agreed with those obtained with the finite element analysis model.

The piezoelectric layers under consideration are composed of PZN-8\%PT. The material's properties ${ }^{14,15,20}$ of the piezoelectric layer and middle layer are listed in Table 1 . The PCTB was built with the following dimensions: $l=40 \mathrm{~mm}$, $h_{p}=0.2 \mathrm{~mm}, h_{m}=0.3 \mathrm{~mm}$ and $w=10 \mathrm{~mm}$.

Figure 3 shows the dynamic responses of the PCTB; including its tip deflection and charge on the electrode, when the piezoelectric bender is driven by voltage or an external tip force $F$. The results of the analysis for the PCTB with large piezoelectric coefficients and EMCCs are shown. The dynamic admittance curve through the theoretical analysis in

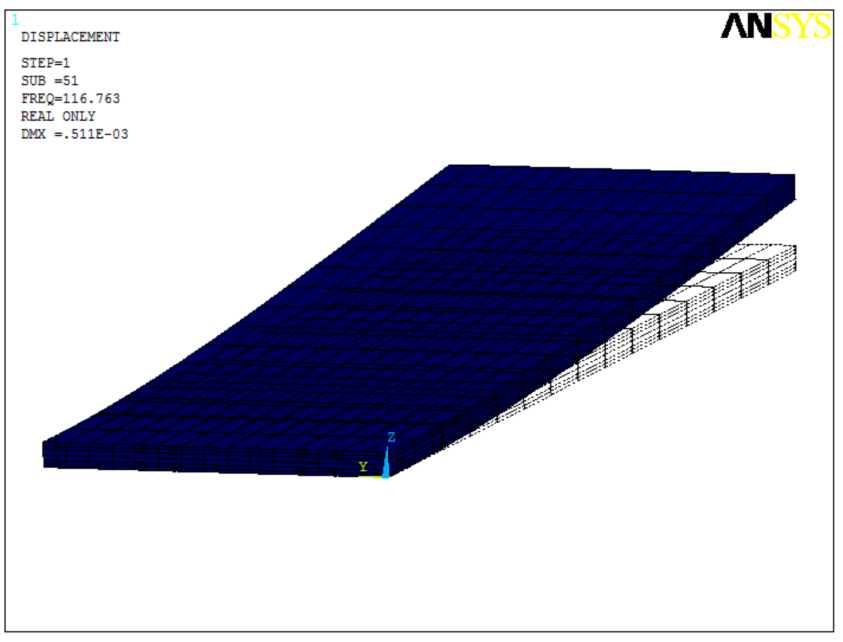

Fig. 2. Simulated shape of the first mode of the symmetrical PCTB.

Table 1. Materials properties used in the calculations.

\begin{tabular}{lccc}
\hline Symbol & Quantity & Value & Units \\
\hline PZN-8\%PT & Piezoelectric & & \\
$d_{31}$ & Piezoelectric strain coefficient & $-1455 \times 10^{-12}$ & C/N \\
$d_{33}$ & & $2890 \times 10^{-12}$ & C/N \\
$d_{15}$ & & $159 \times 10^{-12}$ & C/N \\
$\varepsilon_{11}^{T}$ & Free relative dielectric & 2900 & \\
$\varepsilon_{33}^{T}$ & constant & 7700 & \\
$S_{11}^{E}$ & Elastic compliances constant & $87 \times 10^{-12}$ & $\mathrm{~m}^{2} / \mathrm{N}$ \\
$S_{12}^{E}$ & at short circuit condition & $-13.1 \times 10^{-12}$ & $\mathrm{~m}^{2} / \mathrm{N}$ \\
$S_{13}^{E}$ & & $-70 \times 10^{-12}$ & $\mathrm{~m}^{2} / \mathrm{N}$ \\
$S_{33}^{E}$ & & $141 \times 10^{-12}$ & $\mathrm{~m}^{2} / \mathrm{N}$ \\
$S_{44}^{E}$ & & $15.8 \times 10^{-12}$ & $\mathrm{~m}^{2} / \mathrm{N}$ \\
$S_{66}^{E}$ & & $15.4 \times 10^{-12}$ & $\mathrm{~m}^{2} / \mathrm{N}$ \\
$k_{31}$ & Transverse electromechanical & 0.60 & \\
$\rho$ & coupling factor & & \\
Copper & Density & $8.315 \times 10^{3}$ & $\mathrm{~kg} / \mathrm{m}^{3}$ \\
$E$ & & & \\
$E_{V}$ & Young's modulus & $1.153 \times 10^{11}$ & $\mathrm{~N} / \mathrm{m}^{2}$ \\
$\rho$ & Flexural modulus & $1.388 \times 10^{11}$ & $\mathrm{~N} / \mathrm{m}^{2}$ \\
$\mu$ & Density & $8.96 \times 10^{3}$ & $\mathrm{~kg} / \mathrm{m}^{3}$ \\
\hline & Poisson's ratio & 0.34 & \\
\hline
\end{tabular}




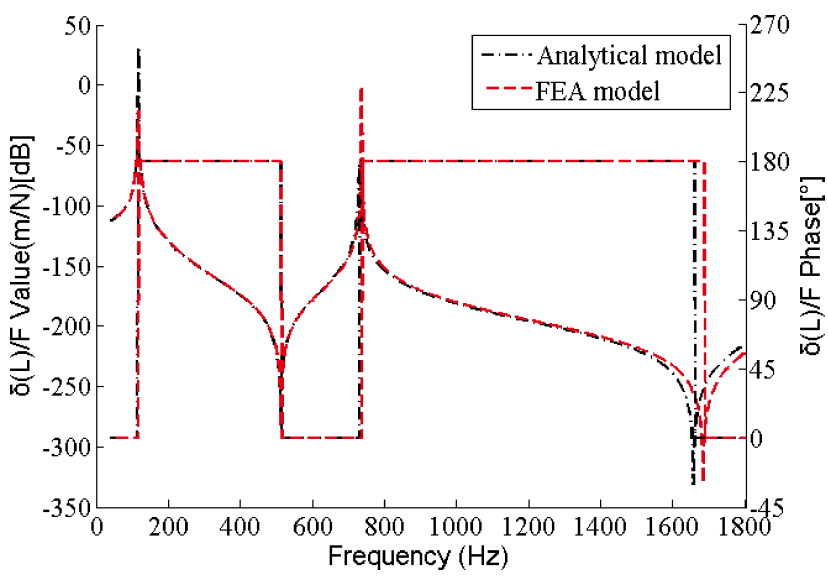

(a)

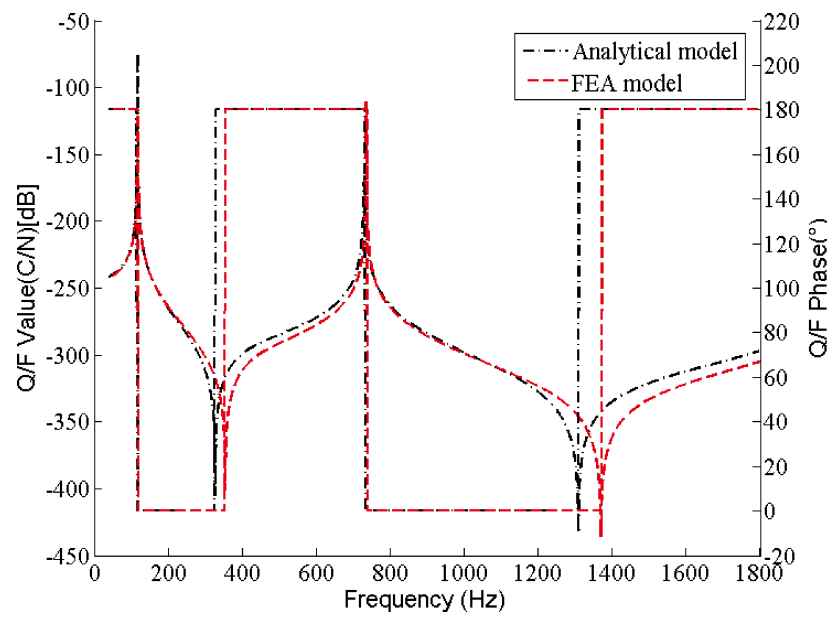

(c)

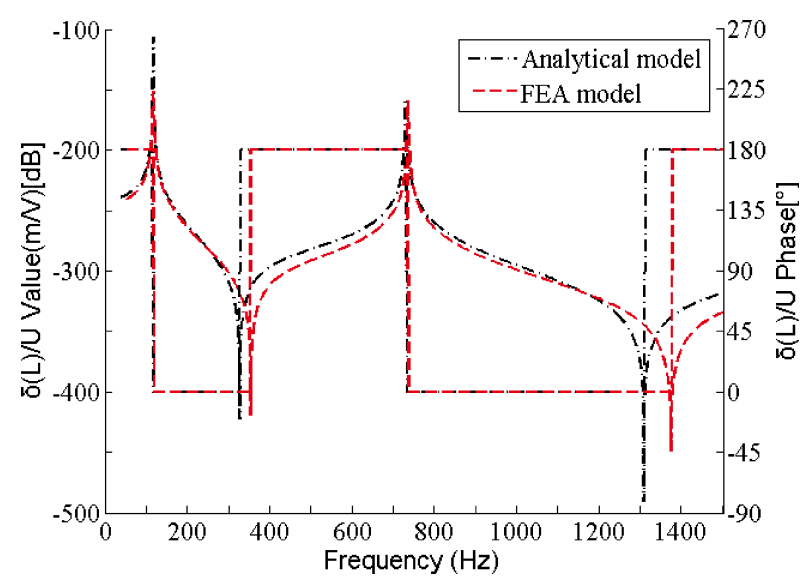

(b)

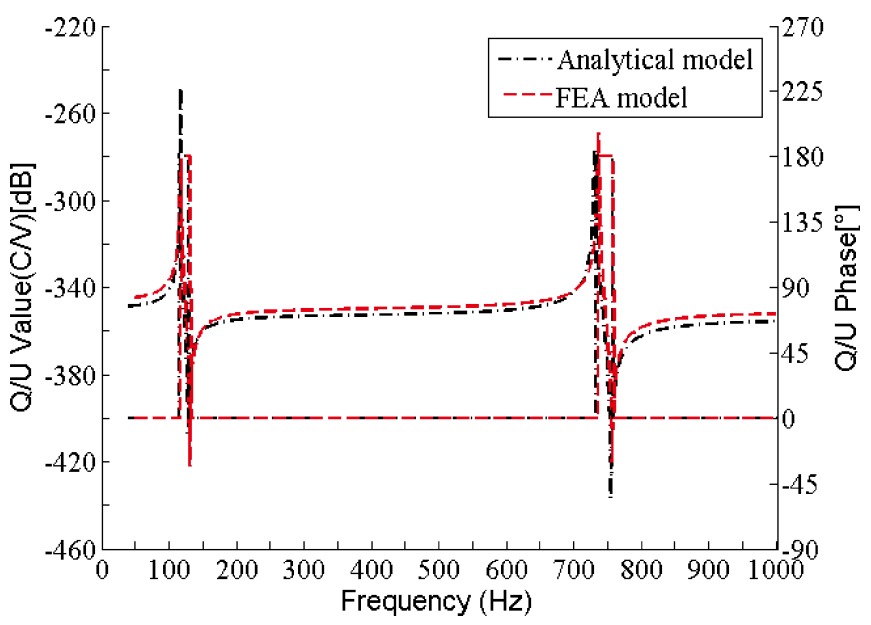

(d)

Fig. 3. The analytical data calculated by the elements in (46) along with finite element method (FEM) simulation data, (a) the (1, 1) element; (b) the $(1,3)$ element; (c) the $(3,1)$ element; (d) the $(3,3)$ element.

terms of the plane strain condition agrees with the results of FEM, as shown in Fig. 3. The resonance frequencies and antiresonance frequencies emerge by turns. After the resonance frequencies appear, the anti-resonance frequencies arise.
These resonant peak frequencies listed in Tables 2 are obtained via admittance model analysis and FEA. $f_{A}$ and $f_{F}$ are the theoretical and FEM analysis flexural resonance and anti-resonance frequencies of the PCTB, respectively;

Table 2. Comparison of resonance and anti-resonance frequencies calculated by the admittance matrix and FEM.

\begin{tabular}{lcccccccc}
\hline & & \multicolumn{3}{c}{ Resonance } & & \multicolumn{3}{c}{ Anti-resonance } \\
\cline { 3 - 5 } Elements & Mode no. & $f_{A}(H z)$ & $f_{F}(H z)$ & $\Delta(\%)$ & $f_{A}(H z)$ & $f_{F}(H z)$ & $\Delta(\%)$ \\
\hline$\delta(\omega) / F(\omega)$ & 1 & 116.7 & 116.7 & 0 & & 512.3 & 512.4 & 0.02 \\
& 2 & 732.1 & 736.5 & 0.6 & 1659 & 1686 & 1.6 \\
$\delta(\omega) / U(\omega)$ & 1 & 116.8 & 116.8 & 0 & & 327.5 & 354.6 & 7.6 \\
& 2 & 730.8 & 736.9 & 0.8 & 1310 & 1375 & 4.7 \\
$Q(\omega) / F(\omega)$ & 1 & 116.7 & 116.7 & 0 & & 327.3 & 354.4 & 7.6 \\
& 2 & 731.8 & 736.5 & 0.6 & & 1311 & 1371 & 4.4 \\
$Q(\omega) / U(\omega)$ & 1 & 116.8 & 116.8 & 0 & & 127.3 & 130.3 & 2.3 \\
& 2 & 730.8 & 736.9 & 0.8 & 754.9 & 756.5 & 0.2 \\
\hline
\end{tabular}


therefore, $\Delta=\left|f_{A}-f_{F}\right| f_{F}$. Theoretical and FEM flexural resonance frequencies of the PCTB are good in agreement with each other. Subtle differences may be attributed to calculation errors during analysis. It is important to note that the above numerical example processes the analytical models as the plane strain condition. The more important reason is probably that ferroelectric single crystals with larger piezoelectric coefficients and $\mathrm{EMCCs}^{14}$ are very easy to lead to larger deformations.

In the last place, the magnitude of the error in the resonant frequency due to neglecting the secondary piezoelectric effect on the flexural rigidity of bender with extraordinarily large piezoelectric coefficients is predicted. To facilitate analysis, the bender height is set to $H=2 h_{p}+h_{m}$, the thickness of the middle layer is set to $h_{m}=\alpha H$, and the thickness of each piezoelectric slice is set to $h_{p}=(1-\alpha) H / 2$. The flexural rigidity of a triple layer bender EI is generally obtained by the transformed cross-section method ${ }^{20,21}$ of the composite beam. Owing to the difference of the flexural rigidity, the error in resonant frequency can be written as

$$
\begin{aligned}
\text { err } & =\frac{\omega_{n}-\omega_{n \_t r}}{\omega_{n}} \\
& \sqrt{4-4 \alpha^{3}+4 \alpha^{3} \eta+\xi(1-\alpha)^{3}} \\
= & \frac{-\sqrt{4-4 \alpha^{3}+4 \alpha^{3} \eta}}{\sqrt{4-4 \alpha^{3}+4 \alpha^{3} \eta+\xi(1-\alpha)^{3}}} .
\end{aligned}
$$

The effective Young's modulus of PZN-8\%PT under plane strain conditions is $11.76 \mathrm{GPa}$, so $\eta=11.802$ and $\xi=3.03$. The result for PZN-8\%PT and copper is presented in Fig. 4. As depicted in Fig. 4, the error depends on the central layer thickness/total thickness ratio. For the symmetric bimorph $(\alpha=0)$, the resonant frequency is theoretically underestimated with an error of $24.6 \%$ for flexural vibration. So, large errors may occur in the analysis of the electromechanical behavior using the conventional model. Notably, after setting $h_{m}=0$, the symmetrical triple-layer structure becomes a symmetric bimorph configuration. The derived

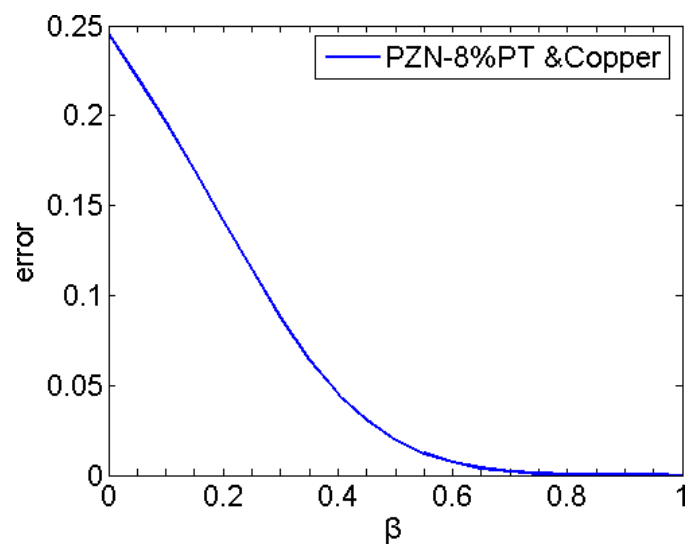

Fig. 4. Error in resonant frequency for the symmetrical piezoelectric bender. models are reduced to those for a symmetrical bimorph cantilever bender.

As one special case of the dynamic admittance, the frequency of vibration $\omega$ approaches zero and the quasi-static transfer admittance can be obtained based on Eqs. (45) and (46). Equations (50) and (51) show the analysis results for the quasi-static transfer admittance. To verify that the derived model is compatible with the existed model of the piezoelectric cantilever bender, the equations have been compared with those reported in Ref. 1 by Wang et al. The analysis in this reference is based on the assumption that no stress but $T_{1}$ is assumed to be zero, namely that it is considered according to plane stress condition. We performed the considerations with the assumption of Wang et al. to compare our results with those in Ref. 1. Comparisons of our results with the equations published by Wang et al. in Ref. 1 show that the elements in the quasi-static admittance matrix are more precise in forecasting quasi-static features for the PCTB with both small and large EMCC than those derived by Wang et al. ${ }^{1}$ due to considering the influence of transverse electromechanical coupling factor $k_{31}$ on the expression of the flexural rigidity EI:

$$
\lim _{\omega \rightarrow 0} Y_{s}(\omega)=\left[\begin{array}{ccc}
\frac{l^{3}}{3 \mathrm{EI}} & \frac{l^{2}}{2 \mathrm{EI}} & \frac{w l^{2}\left(h_{p}+h_{m}\right) d}{4 s^{E} \mathrm{EI}} \\
\frac{l^{2}}{2 \mathrm{EI}} & \frac{l}{\mathrm{EI}} & \frac{w l\left(h_{p}+h_{m}\right) d}{2 s^{E} \mathrm{EI}} \\
\frac{w l^{2}\left(h_{p}+h_{m}\right) d}{4 s^{E} \mathrm{EI}} & \frac{w l\left(h_{p}+h_{m}\right) d}{2 s^{E} \mathrm{EI}} & Y_{s 33}
\end{array}\right],
$$

where $Y_{s 33}=\frac{w l \varepsilon^{T} T}{2 h_{p}}\left(1-k_{31}^{2}+\frac{k_{31}^{2} w h_{p}\left(h_{p}+h_{m}\right)^{2}}{4 s^{E} \mathrm{EI}}\right)$,

$$
\lim _{\omega \rightarrow 0} Y_{p}(\omega)=\left[\begin{array}{ccc}
\frac{l^{3}}{3 \mathrm{EI}} & \frac{l^{2}}{2 \mathrm{EI}} & \frac{w l^{2}\left(h_{p}+h_{m}\right) d}{2 s^{E} \mathrm{EI}} \\
\frac{l^{2}}{2 \mathrm{EI}} & \frac{l}{\mathrm{EI}} & \frac{w l\left(h_{p}+h_{m}\right) d}{s^{E} \mathrm{EI}} \\
\frac{w l^{2}\left(h_{p}+h_{m}\right) d}{2 s^{E} \mathrm{EI}} & \frac{w l\left(h_{p}+h_{m}\right) d}{s^{E} \mathrm{EI}} & Y_{p 33}
\end{array}\right],
$$

where $Y_{p 33}=\frac{2 w l \varepsilon^{T}}{h_{p}}\left(1-k_{31}^{2}+\frac{k_{31}^{2} w h_{p}\left(h_{p}+h_{m}\right)^{2}}{4 s^{E} \mathrm{EI}}\right)$.

Although all the aforementioned discussions do not involve damping, a number of researchers ${ }^{22}$ have used complex material constants with real and imaginary components that refer to an energy loss associated with frequency dependence. So as various forms of damping can be transformed into the equivalent viscous damping, the above matrixes will be available for the realistic system including the damping influence. Therefore, by taking advantage of the elements in the above admittance matrices we can also obtain the various dynamic admittances of the damping system.

\section{Conclusion}

This paper is focused on the dynamic properties of a PCTB. Numerical results coincide with simulations using the FEM. 
These results prove that the analytic models are appropriate descriptions of a symmetrical PCTB with large piezoelectric coefficient and EMCC. However, the assumption in these numerical examples that no deformation is detected in the $y$-direction will need to further be verified with a prototype. To better understand the behavior of symmetrical PCTBs as a function of circular frequency $(\omega), 3 \times 3$ admittance matrices is created considering the impact of the secondary piezoelectric effect on the electric field and the stress field within the piezoelectric material layer. Expressions for the generated deflection, tip angular slope, and electric charge collected by the planar electrodes of PCTBs with large piezoelectric coefficient and EMCC have been presented when PCTBs were excited respectively by an external tip harmonic force $F$, external moment $M$, or applied harmonic voltage $V$. We find the bending rigidity of piezoelectric triple-layer composited bender is affected not by thickness, width and the modulus of elasticity of the different layers but EMCCs of the piezoelectric material; and the larger EMCCs mean more marked effect. Although certain issue has been observed in the existing Cantilever-type piezoelectric devices model, ${ }^{12}$ the launch of the dynamic admittance matrix provides a foundation for the analysis, design, and development of PCTBs with arbitrary EMCCs. They are convenient for the accurate analysis of PCTB structural dynamic properties, including resonance frequency, anti-resonance factor, electrical admittance, induced electric currents.

\section{Acknowledgments}

The authors would like to acknowledge the financial supports from Project 50907042, which was supported by the National Natural Science Foundation of China, and Project 2007DA10512713407 through the Scholarship for Visiting Scholars of State Key Laboratory of Power Transmission Equipment \& System Security and New Technology (Chongqing University).

\section{References}

${ }^{1}$ Q. M. Wang and L. Eric Cross, Constitutive equations of symmetrical triple layer piezoelectric benders, IEEE Trans. Ultrason. Ferroelectr. Freq. Control 46(6) 1343 (1999).

${ }^{2}$ J. K. Park and W. K. Moon, Constitutive relations for piezoelectric benders under various boundary conditions, Sensor. Actuators A-Phys. 117(1) 159 (2005).

${ }^{3}$ J. G. Smits and W. S. Choi, The constituent equations of piezoelectric heterogeneous bimorphs, IEEE Trans. Ultrason. Ferroelectr. Freq. Control 38(3) 256 (1991).
${ }^{4}$ J. G. Smits and A. Ballato, Dynamic admittance matrix of piezoelectric cantilever bimorphs, J. Microelectromech. Syst. 3(3) 105 (1994).

${ }^{5} \mathrm{P}$. Lu and K. Lee, An alternative derivation of dynamic admittance matrix of piezoelectric cantilever bimorph, J. Sound Vib. 266(4) 723 (2003).

${ }^{6}$ J. G. Smits, S. I. Dalke and T. K. Cooney, The constituent equations of piezoelectric bimorphs, Sens. Actuators, A 28(1) 41 (1991).

${ }^{7}$ M. S. Weinberg, Working equations for piezoelectric actuators and sensors, J. Microelectromech. Syst. 8(4) 529 (1999).

${ }^{8}$ A. Erturk and D. J. Inman, Issues in mathematical modeling of piezoelectric energy harvesters, Smart Mater. Struct. 17(6) 065016 (2008).

${ }^{9}$ O. Kursu, A. Kruusing, M. Pudas and T. Rahkonen, Piezoelectric bimorph charge mode force sensor, Sensor. Actuators A-Phys. 153(1) 42 (2009).

${ }^{10} \mathrm{~J}$. Xu and J. Tang, Multi-directional energy harvesting by piezoelectric cantilever-pendulum with internal resonance, Appl. Phys. Lett. 107(21) 213902 (2015).

${ }^{11}$ L. J. Gong, Q. S. Pan and Z. H. Feng, Admittance matrix of a symmetrical triple-layer piezoelectric cantilever, in IEEE 2014 Symp. Piezoelectricity, Acoustic Waves, and Device Applications (SPAWDA) (IEEE, 2014), pp. 322-326.

${ }^{12}$ E. B. Tadmor and G. Kósa, Electromechanical coupling correction for piezoelectric layered beams, J. Microelectromech. Syst. 12(6) 899 (2003).

${ }^{13} \mathrm{~J}$. Peng et al., Orientation dependence of transverse piezoelectric properties of $0.70 \mathrm{~Pb}\left(\mathrm{Mg}_{1 / 3} \mathrm{Nb}_{2 / 3}\right) \mathrm{O}_{3}-0.30 \mathrm{PbTiO}_{3}$ single crystals, Appl. Phys. Lett. 85(25) 6221 (2004).

${ }^{14}$ W. Jiang et al., Characterization of piezoelectric materials with large piezoelectric and electromechanical coupling coefficients, Ultrasonics 41(2) 55 (2003).

${ }^{15} \mathrm{R}$. Zhang et al., Complete set of properties of $0.92 \mathrm{~Pb}$ $\left(\mathrm{Zn}_{1 / 3} \mathrm{Nb}_{2 / 3}\right) \quad \mathrm{O}_{3}-0.08 \quad \mathrm{PbTiO}_{3}$ single crystal with engineered domains, Mater. Lett. 57(7) 1305 (2003).

${ }^{16}$ C. Joh, J. Kim and Y. Roh, Determination of the complex material constants of PMN-28\% PT piezoelectric single crystals, Smart Mater. Struct. 22(12) 125027 (2013).

${ }^{17}$ Standard on Piezoelectricity, IEEE Std. 176-1987 (1988), doi: 10.1109/IEEESTD.1988.79638.

${ }^{18}$ W. P. Mason, Crystal Physics of Interaction Processes, Vol. 23 (Academic Press, 1966).

${ }^{19}$ S. S. Rao, Mechanical Vibrations (Pearson Prentice Hall, 2004), p. 439.

${ }^{20}$ R. C. Hibbeler, Mechanics of Materials (Pearson Education, 2003).

${ }^{21}$ L. J. Gong, J. Q. Li and Y. S. Wang, Analysis of the admittance of symmetrical triple-layer flexural piezoelectric vibrator, in 2011 Int. Symp. Applications of Ferroelectrics (ISAF/PFM) and 2011 Int. Symp. Piezoresponse Force Microscopy and Nanoscale Phenomena in Polar Materials (IEEE, 2011), pp. 1-4.

${ }^{22} \mathrm{D}$. Baral et al., Determination of the primary elastic constants from thin foils having a strong texture, J. Appl. Phys. 53(5) 3552 (1982). 